\title{
M-theory compactifications on hyperbolic spaces
}

\author{
Domenico Orlando \\ Università di Milano-Bicocca and INFN, \\ Sezione di Milano-Bicocca, \\ P.zza della Scienza, 3; \\ I-20126 Milano, Italy
}

\begin{abstract}
Negatively-curved, maximally symmetric hyperbolic spaces enjoy a number of remarkable properties that can be traced back to Riemannian geometry, group theory and algebraic geometry. In this note we recall some such properties and find $H_{n}$ as M-theory solutions.

Based on a talk given at the RTN Workshop 2006, Napoli.
\end{abstract}




\begin{tabular}{c||c|c|c|c|}
$\left(\epsilon_{0}, \epsilon_{n}, \epsilon\right)$ & --- & -++ & -+- & +++ \\
\hline \hline Signature & Minkowski & Minkowski & Euclidean & Euclidean \\
\hline Curvature & - & + & - & + \\
\hline Space & $\mathrm{AdS}_{n}$ & $\mathrm{dS}_{n}$ & $H_{n}$ & $S^{n}$
\end{tabular}

TABLE I: Maximally symmetric spaces

\section{FOREWORD}

Maximally symmetric spaces play an important role in supergravity, because they provide non-trivial, yet manageable solutions. This is essentially because they allow for a natural description not only in terms of Riemannian geometry but also in group theory or algebraic geometry. As a consequence, they enjoy a large number of properties that make them extremely useful as toy models e.g. in cosmology.

Among these spaces, most of the attention has been devoted to anti-de Sitter manifolds and spheres. Here we concentrate our attention on negatively-curved hyperbolic spaces which can be obtained in a more non-trivial manner as M-theory solutions, but have already been considered in literature ([1, 2] $)$.

\section{HYPERBOLIC MANIFOLDS}

Many different definitions can be given for maximally symmetric spaces. The following is perhaps the most intuitive one. An $n$-dimensional maximally symmetric space is a pseudo-sphere in $n+1$ dimensions, i.e. the locus of the points in $\mathbb{R}^{n+1}$ satisfying the equation:

$$
\epsilon_{0}\left(X^{0}\right)^{2}+\left(X^{1}\right)^{2}+\cdots+\left(X^{n-1}\right)^{2}+\epsilon_{n}\left(X^{n}\right)^{2}=\epsilon L^{2},
$$

where the three $\epsilon$ parameters are signs. In particular $\epsilon_{0}$ and $\epsilon_{n}$ specify the signature

of the embedding $\mathbb{R}^{n+1}$ space. There are $2^{3}-2=6$ inequivalent choices for these signs; $(+,+,-)$ gives an empty set and $(-.-,+)$ results in a manifold with two time directions.

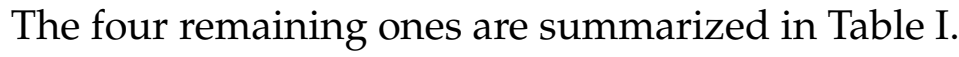

By construction these spaces have constant curvature. In terms of Riemannian geom- 
etry, the relevant tensors can be written as:

$$
R_{a b c d}=\frac{\epsilon}{L^{2}}\left(g_{a d} g_{b c}-g_{a c} g_{b d}\right), \quad R i c_{a b}=R_{a c b}^{c}=\frac{\epsilon}{L^{2}}(n-1) g_{a b}, \quad R=\operatorname{Ric}_{a}^{a}=\frac{\epsilon}{L^{2}} n(n-1) .
$$

From now on we put, without loss of generality, $L=1$.

In the following, we will mainly concentrate on negatively-curved Euclidean manifolds, usually called hyperbolic (Poincarè, Lobachevsky) spaces $H_{n}$.

Another very useful description can be given in terms of Lie groups. A maximally symmetric space is identified with the coset $G / T$ (we quotient with respect to the action of $T$ on the left, $g \sim g t$ ) where $T$ is the maximal subgroup in the group $G$. In particular, one can show that

$$
H_{n}=\frac{S O(1, n)}{S O(n)} \text {. }
$$

The first obvious consequence is that $S O(1, n)$ is the group of isometries of $H_{n}$.

A particularly convenient choice of coordinates, covering the whole manifold, is given by the so-called Poincaré coordinates. The line element takes the form

$$
\mathrm{d} s^{2}=g_{a b} \mathrm{~d} u^{a} \mathrm{~d} u^{b}=\frac{1}{\left(u^{1}\right)^{2}}\left(\left(\mathrm{~d} u^{1}\right)^{2}+\cdots+\left(\mathrm{d} u^{n}\right)^{2}\right),
$$

where $u^{i} \in(0,+\infty)$. In such coordinates it is evident that such manifolds have infinite volume, i.e. the integral $\left(\int \mathrm{d} u^{1} \ldots \mathrm{d} u^{n} \sqrt{g}\right)$ diverges.

Starting from $H_{n}$, it is possible to construct compact manifolds of constant negative curvature by taking the quotient with respect to the action of a freely acting discrete group $\Gamma$. By what we said above, $\Gamma$ must be a discrete subgroup of $S O(1, n)$ (from now on we call this a lattice). It is worth emphasizing that, although $H_{n}$ and $H_{n} / \Gamma$ share the same local properties, the global ones are completely different.

Not only that by choosing a lattice in $S O(1, n)$ one obtains a compact manifold, but it can be shown that $H_{n}$ is the universal cover of any closed manifold $M$ of negative constant curvature. Equivalently, any such $M$ can always be written as $M=H_{n} / \Gamma$ for a suitable choice $\Gamma \subset S O(1, n)$.

One of the most important results in the study of these manifolds is Mostow's rigidity theorem [4]. It states that the geometry of a finite volume hyperbolic manifold of dimension 
greater than two is determined by its fundamental group ${ }^{1}$. More precisely, we can give two equivalent, geometric and algebraic, formulations:

- Given $M$ and $N$, complete, finite-volume hyperbolic $n$-manifolds, if there exists an isomorphism $f: \pi_{1}(M) \rightarrow \pi_{1}(N)$, it is induced by a unique isometry from $M$ to $N$.

- Let $\Gamma_{1}$ and $\Gamma_{2}$ be lattices in $S O(1, n)$ such as $H_{n} / \Gamma_{i}$ has finite volume. If they are isomorphic then they are conjugate.

The theorem is not valid in $d=2$ dimensions. This is compatible with the well known fact that Riemann surfaces of genus $g>1$ (which can always be represented as quotients $\left.H_{2} / \Gamma, \Gamma \subset S O(1,2)\right)$, have a $6(g-1)$-dimensional moduli space.

In view of the following physical applications, we will now concentrate on the lowerdimensional $d=2$ and $d=3$ examples.

$d=2$, or, Riemann surfaces. Depending on their genus, Riemann surfaces can be endowed with a metric that can be spherical (genus $g=0$ ), flat (genus $g=1$ ) or hyperbolic $(g \geq 2)$. This means in particular that any surface of genus $g>1$ can be seen as a quotient $H_{2} / \Gamma$, where $\Gamma$ is a lattice in $S O(1,2)$.

Any surface of genus $g$ can be described in terms of a polygon. In particular we define the metric fundamental polygon as the $(4 g+2)$-gon in which the edges are pairwise identified and the standard fundamental polygon as the $(4 g)$-gon in which the edges are pairwise identified and all the vertices are identified. Both the metric and the standard fundamental polygons describe a surface of genus $g$ as one can easily verify by evaluating the Euler number that in the first case is $\chi=1-(2 g+1)+2=2-2 g$ (if two vertices are independent) and in the second $\chi=1-(2 g)+1=2-2 g$. In the usual notation, an $n$-gon is represented as a string of $n$ letters with exponent \pm 1 depending on the orientation of the edge with respect to an arbitrary positive one. The same letter is used for pairs of identified edges. It is possible to show that the standard fundamental polygon for an oriented Riemann surface of genus $g$ can always be put in the form $A_{1} B_{1} A_{1}^{-1} B_{1}^{-1} \ldots A_{g} B_{g} A_{g}^{-1} B_{g}^{-1}$.

\footnotetext{
${ }^{1}$ In $d=3$ the constaints imposed by Mostow's theorem can be avoided by using Dehn surgery [6], but the price to pay is to use cusped hyperbolic three-manifolds and incomplete metrics. We will not deal with this type of varieties in this context.
} 


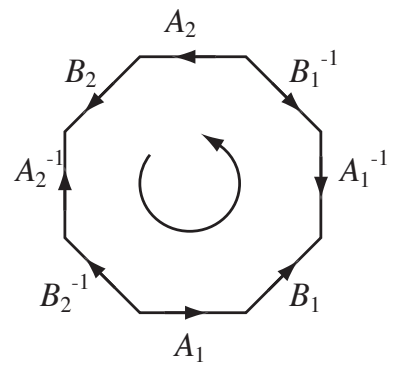

(a)

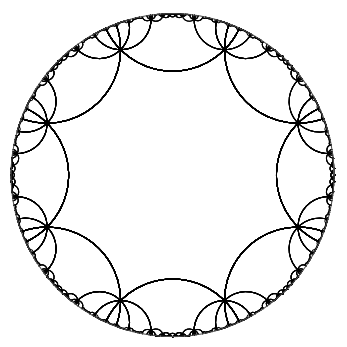

(b)

FIG. 1: Different representations of a Riemann surface of genus $g=2$. (a) Standard fundamental polygon $A_{1} B_{1} A_{1}^{-1} B_{1}^{-1} A_{2} B_{2} A_{2}^{-1} B_{2}^{-1}$; (b) tessellation of the Poincaré disc.

In an equivalent fashion, the fundamental polygons can be seen as elementary cells for a tessellation of the hyperbolic plane $\mathrm{H}_{2}$. In Fig. „ we show one such tessellation, corresponding to a genus two surface, on the Poincaré disc.

Hyperbolic three-manifolds. The three-dimensional case is the first example in which Mostow's theorem holds. In particular, this means that the geometry of a compact hyperbolic three-manifold is fixed by the choice of the lattice and hence by its volume. Here we show how this volume can be computed for a large family of lattices by using algebraic techniques.

The hyperbolic space $H_{3}$ is the coset $S O(1,3) / S O(3)$. This implies that its group of isometries is $S L(2, \mathbb{C}) \sim S O(1,3)$ and in particular any discrete lattice used to compactify is $\Gamma \subset S L(2, \mathbb{C})$. We want to see how the volume of the manifold can be evaluated from the lattice. Let $d$ be a square-free integer (i.e. $d$ does not contain any perfect square as factor). Consider the quadratic field $\mathbb{Q}(\sqrt{-d})=\{a+b \sqrt{-d} \mid a, b \in \mathbb{Q}\}$, i.e. the twodimensional vector space on $Q$ generated by 1 and $\sqrt{-d}$. Let $\mathcal{O}_{d} \subset Q(\sqrt{-d})$ be the ring of integers in this field. Explicitly²:

$$
\mathcal{O}_{d}=\mathbb{Z}[\omega], \quad \omega= \begin{cases}\frac{1}{2}(-1+\sqrt{-d}) & \text { if } d=3 \bmod 4, \\ \sqrt{-d} & \text { otherwise. }\end{cases}
$$

The most simple example is $\mathbb{Q}(\imath)$, the so-called the Gaussian rationals, i.e. the field $\mathbb{Q}(\imath)=\{a+\imath b \mid a, b \in \mathbb{Q}\}$ and $\mathcal{O}_{1}$ is the ring of Gaussian integers $\mathcal{O}_{1}=\mathbb{Z}[\imath]=$

\footnotetext{
${ }^{2}$ When $d=3 \bmod 4, \omega$ is an algebraic integer since it satisfies the equation $\omega^{2}+\omega+(d+1) / 4=0$.
} 
$\{a+\imath b \mid a, b \in \mathbb{Z}\}$.

$\mathcal{O}_{d}$ is a lattice in $\mathbb{C}$, so we can consider the lattice $\operatorname{PSL}\left(2, \mathcal{O}_{d}\right) \subset \operatorname{PSL}(2, \mathbb{C})$ and the compactifications $H_{3} / \Gamma$ where $\Gamma \subset P S L(2, \mathbb{C})$. When one sees $\mathcal{O}_{d}$ as a lattice in $\mathbb{C}$, its area is given by $\operatorname{vol}\left(\mathcal{O}_{d}\right)=\sqrt{D} / 2$ where $D$ is the discriminant:

$$
D\left(\mathcal{O}_{d}\right)= \begin{cases}d & \text { if } d=3 \bmod 4 \\ 4 d & \text { otherwise }\end{cases}
$$

We need now to take into account the difference between considering the lattice on $\mathbb{C}$ and on $\operatorname{PSL}(2, \mathbb{C})$, and this is done by using the formula [5]

$$
\operatorname{vol}\left(H_{3} / \operatorname{PSL}\left(2, \mathcal{O}_{d}\right)\right)=\frac{D^{3 / 2}}{24} \frac{\zeta_{Q}(\sqrt{-d})}{\zeta_{\mathbb{Q}}(2)}
$$

where $\zeta_{Q}(s)$ is the usual Riemann zeta function and $\zeta_{Q}(\sqrt{-d})(s)$ is the Dedekind zeta function on the field $Q(\sqrt{-d})$.

\section{STRING AND M-THEORY BACKGROUNDS}

In this section, we describe the most simple configuration in which hyperbolic spaces can appear as M-theory backgrounds. Before starting, it is worth remarking that negatively-curvature Euclidean spaces are not 'natural' backgrounds. Looking at the structure of the equations of motion, in fact, one would expect, in the presence of gauge fields and without arbitrary cosmological constant, to find negative-curvature Minkowski spaces (anti-de Sitter) or positive-curvature Euclidean space (spheres). And, indeed, a no-go theorem exists for positive-curvature Minkowski spaces (de Sitter) [3].

Consider the following ansatz for the metric and gauge fields in eleven dimensional supergravity:

$$
\left\{\begin{array}{l}
M_{11}=M^{(1)} \times M^{(2)} \times M^{(3)}, \\
F_{[4]}=Q \omega^{(3)},
\end{array}\right.
$$

where $M^{(i)}$ are maximally symmetric spaces of dimension $d_{i}=\operatorname{dim} M^{(i)}$, with the constraint $d_{3}=4, M^{(1)}$ has $(-,+, \ldots,+)$ signature, $\omega^{(3)}$ is the volume form for $M^{(3)}$ and $Q$ is a constant. First of all, we remark that the Chern-Simons contribution to the action $F_{[4]} \wedge F_{[4]} \wedge C_{[4]}$ is vanishing, and that $\mathrm{d} F_{[4]}=0$ since by definition $\omega^{(3)}$ is covariantly 
constant. We must then solve only the Einstein equation:

$$
R_{\mu \nu}-\frac{1}{2} R g_{\mu \nu}-\frac{1}{2}\left|F_{[4]}\right|_{\mu \nu}^{2}+\frac{1}{4}\left|F_{[4]}\right|^{2} g_{\mu \nu}=0,
$$

where with the notation $\left|F_{[p]}\right|^{2}$ we mean $\left|F_{[p]}\right|_{\mu v}^{2}=\frac{1}{p !} F_{\mu \mu_{2} \ldots \mu_{p}} F_{v}{ }^{\mu_{2} \ldots \mu_{p}}$.

Since the $M^{(i)}$ 's are symmetric spaces, we can split the Ricci tensor in blocks and each block will be proportional to the corresponding metric. To fix the notation we can introduce the parameters $k_{i}$ as

$$
\left.R_{\mu v}\right|_{i}=\left.k_{i} g_{\mu v}\right|_{i}, \quad i=1,2,3,
$$

so that the Ricci scalars are given by $R_{i}=k_{i} d_{i}$ and the overall curvature is $R=\sum_{i} k_{i} d_{i}$.

The gauge field is proportional to the volume form, so it is easy to verify that its square is proportional to the metric:

$$
\left|F_{[4]}\right|_{a b}^{2}=Q^{2} g_{a b}
$$

where $a$ and $b$ only run over the four coordinates of the $M^{(3)}$ manifold.

We hence find that the equations of motion take the form of an algebraic system for the $k_{i}^{\prime}$ s:

$$
2 k_{i}-R=-\left(1-2 \delta_{2, i}\right) Q^{2}, \quad \text { for } i=1,2,3,
$$

where $\delta_{2, i}$ is the Kronecker delta. Before solving the system it is worth to make two remarks that would remain true also for more general ans ̈̈ze of the same type:

- Having a minus sign on the RHS implies that negative values for $k_{i}$ are possible.

- Summing the equations for the $k_{i}$ one finds that the curvature of the Minkowski manifold $M^{(1)}$ is always negative. Or, in other words, one cannot obtain de Sitter compactifications in this framework.

Solving for $k_{i}$ we find:

$$
k_{0}=k_{1}=-\frac{Q^{2}}{3}, \quad k_{2}=\frac{2 Q^{2}}{3}
$$

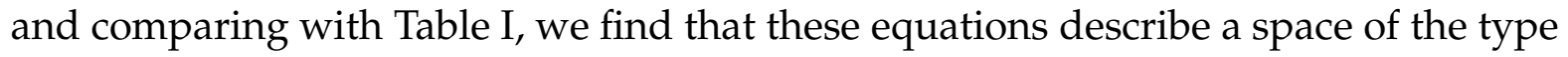

$$
M_{11}=\mathrm{AdS}_{d} \times H_{7-d} \times S^{4}
$$


The system in Eq. (12) only depends on the local properties of the space and remains valid if instead of $H_{7-d}$ we consider a $(7-d)$-dimensional compact hyperbolic manifold $H_{7-d} / \Gamma$. In this way we find that the solution above also describes an $\mathrm{AdS}_{d}$ compactification on a direct product $H_{7-d} / \Gamma \times S^{4}$ which is particularly interesting in the $d=4$ and $d=5$ cases.

The next natural step is to study the stability properties of the solutions we outlined. This goes beyond the scope of the present note. We will however remark that even if maximally symmetric spaces have vanishing Weyl tensor and so preserve part of the supersymmetry, this property will in general be lost in the case of discrete quotients $H_{n} / \Gamma$. The existence of Killing spinors compatible with the boundary conditions has to be checked for each choice of the lattice $\Gamma$.

The author thanks C. Bachas, I. Bakas, E. Cremmer, Y. Dolivet, D. Freedman, C. Kounnas, M. Petropoulos, M. Porrati and S. Stieberger for stimulating scientific discussions. The author is supported in part by INFN and MIUR under contract 2005-024045 and by the European Community's Human Potential Program MRTN-CT-2004-005104.

[1] Jerome P. Gauntlett, Nakwoo Kim, and Daniel Waldram. M-fivebranes wrapped on supersymmetric cycles. Phys. Rev., D63:126001, 2001.

[2] A. Kehagias and J. G. Russo. Hyperbolic spaces in string and m-theory. JHEP, 07:027, 2000.

[3] Juan M. Maldacena and Carlos Nunez. Supergravity description of field theories on curved manifolds and a no go theorem. Int. J. Mod. Phys., A16:822-855, 2001.

[4] G.D. Mostow. Quasi-conformal mappings in n-space and the rigidity of the hyperbolic space forms. Publ. Math. IHES, 34:53-104, 1968.

[5] P. Sarnak. The arithmetic and geometry of some hyperbolic three manifolds. Acta Mathematica, 151(1):253-295, 1983.

[6] W. Thurston. The Geometry and Topology of Three Manifolds. Princeton Un. Lecture Notes, 1978. 